\title{
Empirical Research on Chinese Warrants Market Based on the Montecarlo Pricing Options Under Levy Process
}

\author{
Li Zhou ${ }^{1}$, Hong Zhang ${ }^{1}$, Jian Guo ${ }^{1}$, Anjie Deng ${ }^{2}$ \\ ${ }^{1}$ School of Information, Beijing Wuzi University, Beijing, China \\ ${ }^{2}$ School of Banking and Finance, University of International Business and Economic, Beijing, China
}

Email address:

dr.yuwenjunxian@gmail.com (Hong Zhang)

\section{To cite this article:}

Li Zhou, Hong Zhang, Jian Guo, Anjie Deng. Empirical Research on Chinese Warrants Market Based on the Montecarlo Pricing Options Under Levy Process. American Journal of Applied Mathematics. Vol. 3, No. 3, 2015, pp. 129-137. doi: 10.11648/j.ajam.20150303.19

\begin{abstract}
After the 2008 financial crisis, the global derivatives trading volume in options proportion is growing, more and more investors build portfolios using options to hedge or arbitrage, our futures and stock options will soon open. Theoretical research of options is also changing, option pricing models under Levy processes developed rapidly. In this context, a review of the China's warrants market and the introduction of option pricing models can not only help us to reflect Chinese financial derivatives market regulation, but also to explore the option pricing theory for China's financial market environment. In the framework of Monte Carlo simulation pricing, we established mufti-Levy process option pricing models, the structural model for the given parameter estimation and risk-neutral adjustment method are discussed, the last part of this chapter is an empirical analysis of China warrants trading data in order to prove the validate of Levy models.
\end{abstract}

Keywords: Levy Stochastic Processes, Option Pricing Models, Chinese warrants Market, American Option Pricing, Risk-Neutral Adjustment, Variance Reduction Techniques

\section{Introduction}

From the financial series statistical characteristics can be seen, although the normal random number can be more effective to describe the real data, but the tail distribution of financial data is still significantly deviate from the normal distribution, and the classical model can not effectively describe the volatility of financial asset rate time-varying characteristics, these problems are the classic option pricing model cannot overcome. Based on these reasons, this chapter introduces the option pricing model of Levy random distribution correction, using empirical Chinese warrants market data, compared with the Black-Scholes-Merton model, verify the validity of the model.

The pricing model of all using Monte Carlo simulation method, this method mainly depends on the pricing effect to the following factors: first, the Monte Carlo simulation method for random path based on the assets of simulating and calculating the derivatives pricing model, so the theory of basic assets is an accurate representation of the statistical characteristics of the real historical data has a decisive effect on the accuracy of the final the results, one of which is the focus of this research, this paper mainly uses the Levy model based on random number model. Secondly, in the historical data conditions, estimation of model parameters is an important step, to ensure the accuracy of the estimation, but also to ensure the efficiency and robustness of the integral method. Third, the risk neutral measure conversion is an indispensable process in the middle, but due to different market efficiency difference, pmeasure transformation effect is also different. The fourth is a random simulation of this step; generate Levy random number is necessary to meet the assumption of distribution, but also to improve the overall efficiency of simulated pricing.

\section{The Empirical Results of Chinese Warrants Market}

According to exercise direction, we can divide share warrants into put warrants and warrants. According to warrants issued, it can be divided into equity warrants and covered warrants. According to the execution mode of warrants, warrants can also be divided into European, American and Bermuda Warrants. If it is the equity warrants, the newly issued shares would "dilute" the existing net assets of equity when the warrant is executed .In accordance with this effect, the pricing formula for the European Warrants is: 


$$
C_{t}^{\text {call }}=e^{-r(T-t)} E^{Q}\left[\operatorname{Max}\left(\rho\left(\frac{N_{S} S_{T}+N_{C} \rho K}{N_{S}+N_{C} \rho}-K\right), 0\right) \mid F_{t}\right]
$$

$N_{S}$ and $N_{C}$ denote respectively the number of underlying stocks which is still in circulation at the date of implementation and the number of warrants.

$\rho$ is the ratio for the exercise of warrants, $\mathrm{K}$ is the exercise price of warrants, $S_{T}$ stands for the stock price at the date of exercise. "Dilution effect" is mainly reflected in the following two aspects. First, the issuers of equity warrants will get $C_{0}^{\text {call }} N_{C}$ at the issue date of warrants and the listed companies can get cash flow $N_{C} \rho K$ through issuing additional shares with the exercise price at the exercise date of warrants. The number of Company's share capital after the exercise will become $N_{S}+N_{C} \rho$. We have considered that the cash flow $C_{0}^{\text {call }} N_{C}$ which is generated by the issue date of warrants has been passed to the market through the financial statements and reflected in the stock price $\mathrm{S}$ finally before the vesting date T's arrival. So this section is no longer listed separately in equation (1). Moreover, at the time of the issue of equity warrants, the generated cash flow is a low proportion of the total market capitalization. So this simplification will not cause a significant impact. Considering warrants with cash settlement does not have "dilution" effect, the pricing formula for its European Warrants is:

$$
\begin{aligned}
& C_{t}^{\text {call }}=e^{-r(T-t)} E^{Q}\left[\operatorname{Max}\left(\rho\left(\hat{S}_{T}-K_{T}\right), 0\right) \mid F_{t}\right] \\
& C_{t}^{\text {put }}=e^{-r(T-t)} E^{Q}\left[\operatorname{Max}\left(\rho\left(K_{T}-\hat{S}_{T}\right), 0\right) \mid F_{t}\right]
\end{aligned}
$$

\subsection{The Data Statistical Description}

From 2005 to 2009 , in order to complete the share reform of listed companies, the stock exchanges in Shanghai and Shenzhen listed a total of 55 warrants traded contract, including 26 covered warrants and 29 equity warrants and the exercisable way includes continental, Bermuda and American. In this article, we only price the European warrants. To contrast the warrants horizontally, we set the pricing date of all warrants 200 days before the date of implementation. At the same time, we removed the raw water CTP1 whose time of trading is less than 200 days and CWB 1 which is unstable under the G7R-GARCH model and priced the remaining 38 warrants. The reasons why we choose that many warrants are as follows. During the four years' existence of warrants market, the market is in a speculative atmosphere in the whole and the market price is off the normal value for a long time, but each period and each class have its own warrants feature, so here we choose most of the warrants in the last inter-sample comparison, trying to analyze the warrants market conditions more carefully. Since the small number of contracts of the warrants market, we need to take as many samples in order to increase the robustness and reliability of the results. The following table is the basic information of these warrants:

Table 1. China warrants market European warrants basic information.

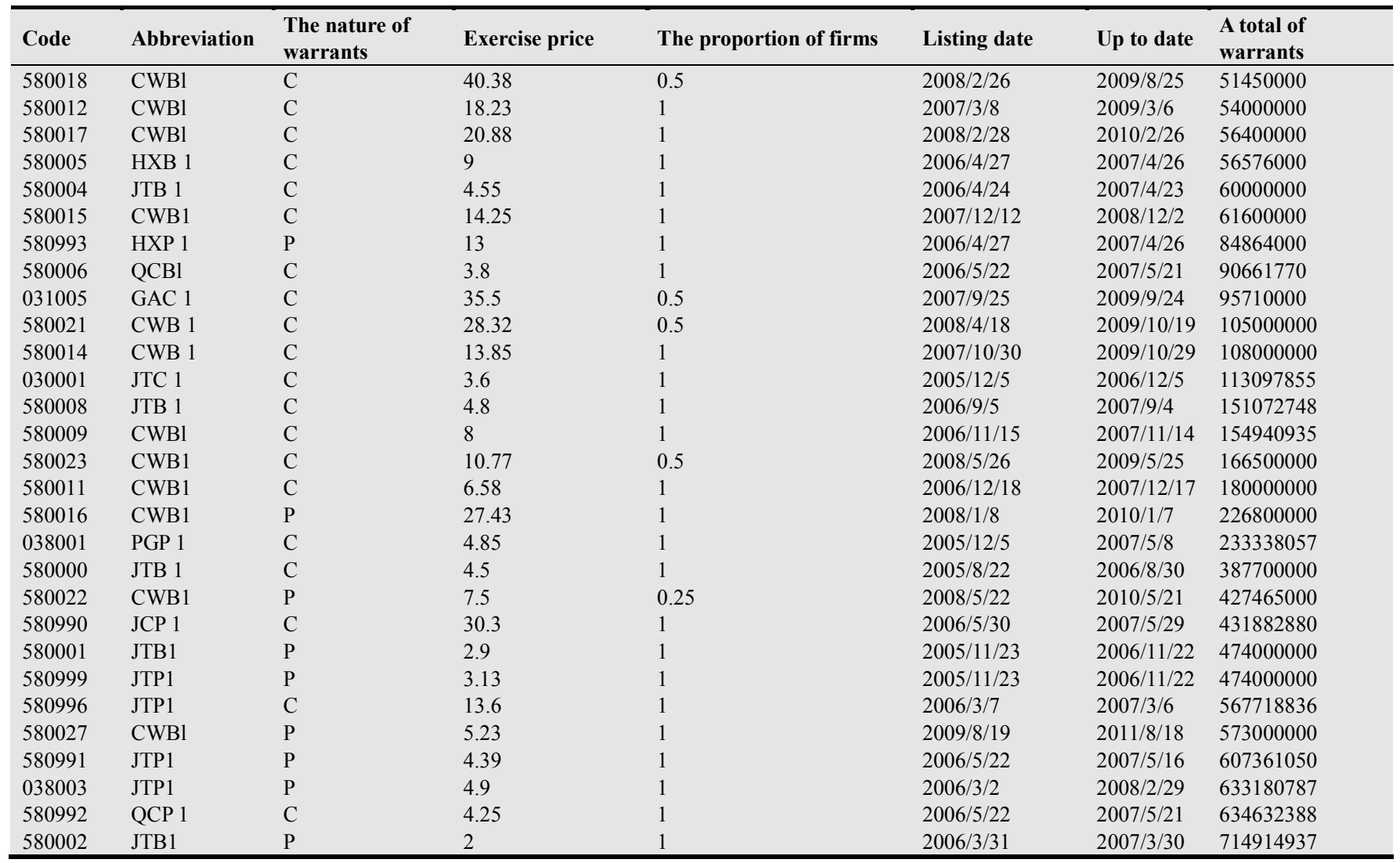




\begin{tabular}{|c|c|c|c|c|c|c|c|}
\hline Code & Abbreviation & $\begin{array}{l}\text { The nature of } \\
\text { warrants }\end{array}$ & Exercise price & The proportion of firms & Listing date & Up to date & $\begin{array}{l}\text { A total of } \\
\text { warrants } \\
\end{array}$ \\
\hline 580995 & JTP1 & $\mathrm{C}$ & 2.45 & 1 & $2006 / 3 / 31$ & $2007 / 3 / 30$ & 714914937 \\
\hline 580013 & CWB 1 & $\mathrm{C}$ & 10.2 & 1 & $2007 / 4 / 17$ & $2009 / 4 / 16$ & 727500000 \\
\hline 580003 & JTB1 & $\mathrm{C}$ & 2.8 & 1 & $2006 / 4 / 7$ & $2007 / 4 / 4$ & 925705299 \\
\hline 580007 & CWB1 & $\mathrm{C}$ & 5.5 & 1 & $2006 / 5 / 25$ & $2007 / 5 / 24$ & 1228010640 \\
\hline 580010 & CWB1 & $\mathrm{C}$ & 3.4 & 1 & $2006 / 11 / 29$ & $2008 / 11 / 28$ & 1265000000 \\
\hline 580989 & JTP1 & $\mathrm{P}$ & 7.43 & 0.5 & $2007 / 6 / 21$ & $2008 / 6 / 20$ & 1400000000 \\
\hline 580026 & CWBl & $\mathrm{C}$ & 15.44 & 1 & $2008 / 10 / 10$ & $2010 / 10 / 9$ & 1761200000 \\
\hline 580997 & CMP 1 & $\mathrm{P}$ & 5.65 & 0.5 & $2006 / 3 / 2$ & $2007 / 8 / 31$ & 2241336679 \\
\hline
\end{tabular}

Seen from the table, our warrants market has more warrants than put warrants, which is consistent with the background of the split-share reform. The time of circulation is from the beginning of August 2005 when JTB 1 listed first to August 2011 when CWB 1 ended the exercise. During the six-year deal, the variety and number of contracts of our warrants market trading are very scarce compared to mature foreign exchange market warrants or options, but the trading volume and handover rate is very high, far beyond the normal level. For the specific content, you can refer to the empirical articles by Yuan et al. (2007), and Xiong and Yu (2011).

\subsection{Levy Option Pricing Model Parameter Estimation Results}

Before performing a Monte Carlo simulation, the first thing to do is to use historical data to test the unknown parameters in the Levy model. Comparing NIG and VG process with the normal distribution can better describe high-order moment features of the data, but its distribution function is very complex. After it is combined with biased GARCH model, there will be a lot of parameters to be estimated and the model forms are complex. Therefore, the operational efficiency is very low using the traditional maximum likelihood estimation. But the form of moment conditions in Levy process is relatively simple, measurement methods under GMM framework can be used for parameter estimation. Carrasco et al. (2007) proposed GMM with a continuum of moment condition which is a good solution to the problem of parameter estimation Levy. So we use GMM model for the parameter estimation.

This model focuses on the volatility of financial data, skewness and kurtosis's impact on derivatives pricing, so firstly, build analytic vectors “ $\bar{m}=\left[m_{1}(\theta), m_{2}(\theta), m_{3}(\theta), m_{4}(\theta)\right]$ " from the first moment to the fourth moment, where $m_{i}(\theta)$ is the analytical formula of the i-th moment, $\theta=\left\{\theta_{R}, \theta_{h}, \theta_{D}\right\}$ is the parameter to be estimated including the average term parameter of GARCH model, the variance term parameters and random item parameter e of Levy distribution. Compute sample moments $\bar{m}=\left[\bar{m}_{1}, \bar{m}_{2}, \bar{m}_{3}, \bar{m}_{4}\right]$ after obtaining explicit expression vector of population moments. Estimate parameters by optimizing the distance between moment vector and sample moments of the vector, in which $\mathrm{W}$ is a diagonal matrix, used to re-empowerment the objective function.

$$
\hat{\theta}=\arg \min _{\theta}\left\{[\bar{m}(\theta)-m]^{\prime} W^{-1}[\bar{m}(\theta)-m]\right\}
$$

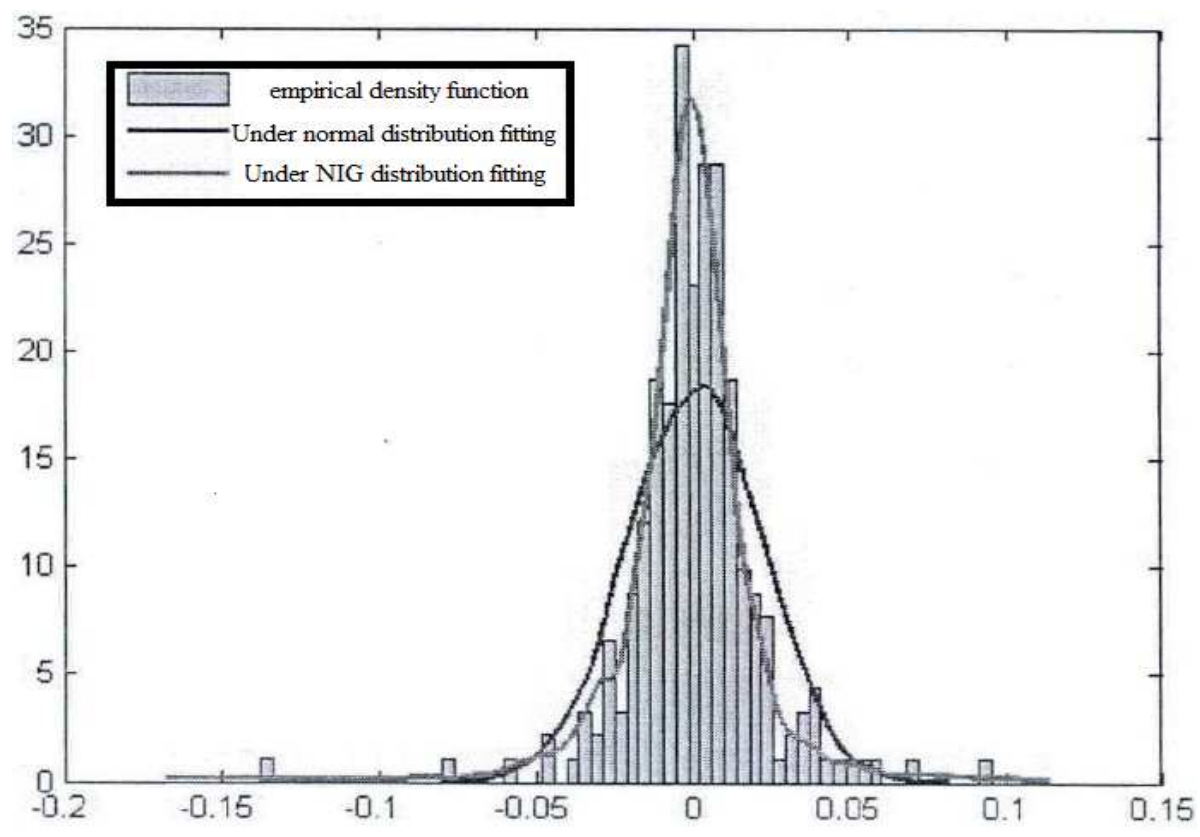

Fig. 1. The fitting effect of NIG distribution and the empirical distribution. 


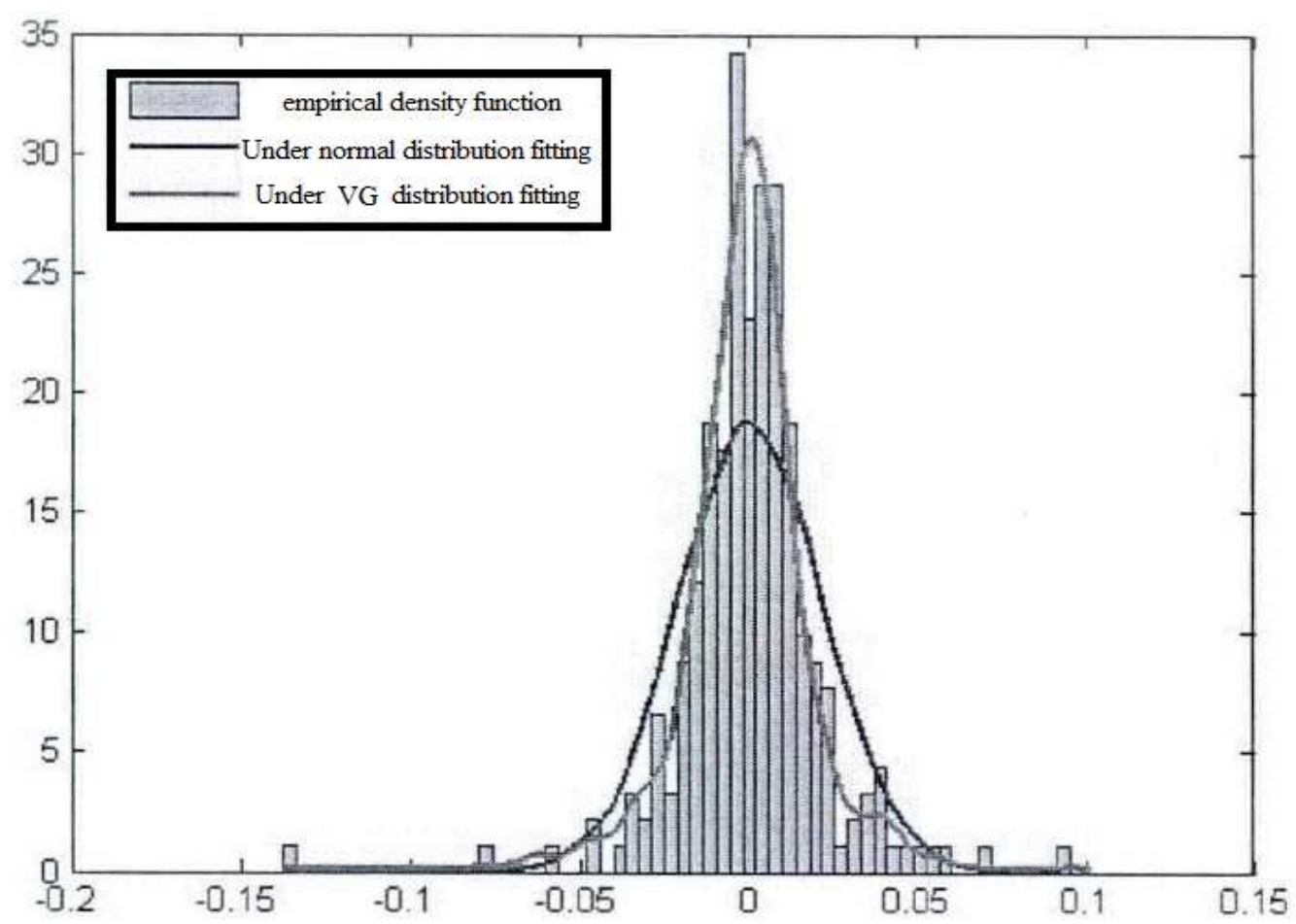

Fig. 2. The fitting effect of $V G$ distribution and the empirical distribution.

Due to a larger number of warrants pricing, the results of parameter estimation are shown in Appendix 4. Select JTB1 data of 200 days from January 11, 2006 to November 14, 2006 and extract a logarithmic rate of return. Then get the figure 1 by using geometric Brownian motion, NIG distribution and VG distribution to estimate and simulate parameters. By contrast, we can see that both of the Levy distribution models can simulate the real data features of "fat tail". The fitting effect of skewness is also much better than the normal distribution, reflecting the advantage that Levy distribution has in the financial Asset Pricing model.

\subsection{The Simulation Results of Levy Option Pricing Model}

After obtaining the results of Levy option model parameters, the next thing to do is to price option model. The three main steps are as follows. First, adjust the risk-neutral of the parameters. Second, generate a corresponding random number path. Finally, do the Monte Carlo simulation pricing based on the black-scholes pricing formula.

The model used in this chapter is relatively simple. Supposing rate of return variables of stocks $\varepsilon_{t}=\ln \left(S_{t}\right)-\ln \left(S_{t-1}\right) \quad$ subject to the corresponding Levy process, just adjust the parameters mean. If the risk-free return on assets is given, introduce a correction value adj $=r-\eta(-i)$, where $\mathrm{R}$ is the risk-free rate of return and $\mathrm{r}(\mathrm{u})$ is the random path generated by random algorithm, based on the parameter deduction of the difference value of feature index and the drift. After generating a sequence of rate of return, we can get the stock price simulation sequence from the first phase to iterate, based on the simulation sequence and initial conditions of asset prices. The iterative method is $\hat{S}_{t}=\hat{S}_{t-1} \exp \left(\hat{\varepsilon}_{t}\right)$. Then simulate the results of options in accordance with European option pricing:

$$
\begin{aligned}
& \hat{C}_{t}^{\text {call }}=e^{-r(T-t)} E^{Q}\left[\operatorname{Max}\left(\rho\left(\hat{S}_{T}-K_{T}\right), 0\right) \mid F_{t}\right] \\
& \hat{C}_{t}^{\text {put }}=e^{-r(T-t)} E^{Q}\left[\operatorname{Max}\left(\rho\left(K_{T}-\hat{S}_{T}\right), 0\right) \mid F_{t}\right]
\end{aligned}
$$

Every time you simulate a path, you can get the simulation result of an option. After 100,000 simulations, the simulation results convergence has been able to meet the requirements and get an estimate of the option by calculating the average of all simulation samples.

The main purpose of this section is to show how the general Levy stochastic model can be applied to the Monte Carlo simulation option pricing. So the model used Levy random process directly with the Warrant data and the warrants pricing formula (3115).It is used for pricing all European Options in NIG, VG, geometric Brownian motion, these three stochastic models. Pricing results are displayed in Appendix 1. In order to ensure the credibility of the result of comparison between models, all 38 European warrants have been priced. Each warrant pricing result has different accuracy sort. Roughly, nonnormality Levy random has advantage over geometric Brownian motion. In order to have a more accurate comparison result statistically, four statistical indicators are introduced to do the error calculation. They are RMSE (root mean square error), RMSE\% (percentage of RMSE), AAE (average absolute error) AAE\% (percentage of AAE). The methods for constructing these indicators are as follows. 


$$
\begin{aligned}
& R M S E=\sqrt{\sum_{i=1}^{N} \frac{\left(C_{i}^{\text {Model }}-C_{i}^{\text {Maket }}\right)^{2}}{N}} \\
& R M S R E=\sqrt{\sum_{i=1}^{N} \frac{\left(C_{i}^{\text {Model }}-C_{i}^{\text {Maket }}\right)^{2}}{N \times\left(C_{i}^{\text {Maket }}\right)^{2}}} \\
& A A E=\frac{\sum_{i=1}^{N}\left|C_{i}^{\text {Model }}-C_{i}^{\text {Maket }}\right|}{N} \\
& A A R E=\frac{\sum_{i=1}^{N}\left|C_{i}^{\text {Model }}-C_{i}^{\text {Maket }}\right|}{C_{i}^{\text {Maket }} \cdot N}
\end{aligned}
$$

Among them there are $\mathrm{N}$ authority card, $C_{i}^{\text {Maket }}$ said the warrants market price, $C_{i}^{\text {Model }}$ said Model to simulate the price accordingly. RMSE and AAE error absolute index of two indicators, and RMSE \% \% and AAE is two relative indexes. Bringing in appendix 1 estimate results to the four index calculation formula, get pricing error statistics:

Table 2. Levy stochastic model pricing errors.

\begin{tabular}{llll}
\hline & BS & NIG & VG \\
\hline RMSE & 2.440484 & 1.97283 & 1.940419 \\
RMSE\% & 0.650607 & 0.519803 & 0.520819 \\
AAE & 1.727636 & 1.3935 & 1.379764 \\
AAE $\%$ & 0.561923 & 0.449254 & 0.449426 \\
\hline
\end{tabular}

BS in the table represents the Black - Scholes - Merton model calculation of authority card price;

NIG and VG respectively represent two types of Levy of warrants pricing under random distribution results

It can be seen from the pricing results in Appendix 5 and the pricing errors in Table 2 that not all models can accurately reflect the market price of the warrants. For example, the price of warrants "580023" CWB 1 is 0.007447 yuan according to Black-Scholes-Merton model. Considering its market price of 35.40 yuan at that day and a call option exercise price of 10.77 yuan, 580023 is a deep OTM warrants and the price should be very close to 0 yuan. So the index of Black-Scholes-Merton model has a certain rationality. But if we analyze the previous volatility Combibloc stock, we will find its fourth moment is very high. It is a warrant which has a strong jumping feature. When these factors are taken into account, the stock index is 0.513709 yuan according to NIG model and the price is 0.506973 yuan according to VG model, which proves that the correction for normal distribution in Levy model has a strong impact on this stock. In fact, the price of this warrant has been 2.53 yuan, much larger than the prices of the few theoretical models. There are many similar examples of big pricing errors. We can also find that the order of these several pricing models is not determined. In the example of warrant "580,999",the pricing result of NIG model is closest to the market price, better than VG model, while BS model has the biggest bias. But for the warrant "580,991", the pricing accuracy of VG model is slightly higher than NIG model. These results suggest that the accuracy of warrant pricing in the Levy process is not always the same. There is no Levy stochastic model applicable to all data which demonstrates the randomness of Monte Carlo simulation pricing.

But overall, after using NIG and VG model of Levy random distribution, pricing results are much better than traditional Black-Scholes-Merton model. It can be seen from Table 2's conclusion that the percentage of RMSE\% and $\mathrm{AAE} \%$ have improved by $10 \%$. The result proves that considering jumping characteristic of the stochastic process can greatly improve pricing accuracy, but all the pricing results have great biases, limited by the foam characteristics of China warrants market. The result is consistent with Xiong and Yu (2011)'s study. In this chapter, the Levy random distribution is introduced completely to the Monte Carlo simulation pricing of options based on the basic theory of option pricing. It is the core chapter of the text.

\section{Summary}

Levy process generally refers to random numbers classes which meet three characteristics. Currently, a lot of Levy random number models can be used to describe the statistical characteristics of financial data. Derivatives pricing models are basically based on no-arbitrage assumption. Although this assumption is too strict for a lot of markets, there is still no better choice in academia. The simulation of this paper is also based on this assumption. Technology of random number simulation is the basis of Monte Carlo simulation techniques. Only with the effective and stable algorithm of random number generation, stochastic models of Levy distribution can get the exact numerical solution, or the model cannot be effectively verified and will not be used for option pricing. In 3.6, empirical researches of China warrants are done. In order to ensure the robustness of the results, I picked up almost all of the European warrants and priced them in VG stochastic model and NIG model, compared with the traditional Black-Scholes-Merton model. Pricing results are as follows. 1. By comparison, it can be seen that the accuracy of VG and NIG model is significantly higher than traditional Black-Scholes-Merton model because VG and NIG process can better describe the jumping characteristics of China's stock market. 2. The pricing error between the two models is small, confirming the high robustness of the algorithm used in this section from the side and the practicability of the relevant models and methods. 3. The trading price of our warrants market deviates significantly from its intrinsic value. There is a severe bubble. The results show that the theoretical model the West set up on the basis of no-arbitrage assumption cannot be applied to the Chinese market. The results also verify the view some scholars hold that there is excessive speculation in our warrant market.

\section{Acknowledgements}

This paper is funded by the project of National Natural Science Fund, Logistics distribution of artificial order picking 
random process model analysis and research(Project number: 71371033); and funded by intelligent logistics system Beijing Key Laboratory (No.BZ0211); and funded by scientific-research bases--- Science \& Technology Innovation Platform---Modern logistics information and control technology research (Project number: PXM2015_014214_000001); and funded by 2014-2015 school year, Beijing Wuzi University, College students' scientific research and entrepreneurial action plan project (No.68); and funded by Beijing Wuzi University, Yunhe scholars program (00610303/007); and funded by Beijing Wuzi University, Management science and engineering Professional group of construction projects. (No. PXM2015_014214_000039). University Cultivation Fund Project of 2014-Research on Congestion Model and algorithm of picking system in distribution center (0541502703)

\section{Appendix}

Table 3. The results of NIG-GJR-GARCH pricing model.

\begin{tabular}{|c|c|c|c|c|}
\hline ‘code & real & Levy_GARCH & Levy & BS \\
\hline 580999 & 0.698 & 0.640831 & 0.556481 & 0.525278 \\
\hline 580997 & 0.475 & 0.151189 & 0.100026 & 0.008904 \\
\hline 580996 & 0.915 & 1.334142 & 1.220966 & 1.303878 \\
\hline 580995 & 0.574 & 0.440372 & 0.401638 & 0.37326 \\
\hline 580994 & 1.281 & 0.740764 & 0.660899 & 0.484467 \\
\hline 580993 & 1.371 & 0.708849 & 0.684944 & 0.563156 \\
\hline 580992 & 0.642 & 0.323328 & 0.329755 & 0.240395 \\
\hline 580991 & 0.72 & 0.695037 & 0.632059 & 0.625238 \\
\hline 580990 & 1.073 & 0.478697 & 0.396746 & 0.231048 \\
\hline 580989 & 1.526 & 0.679302 & 0.401881 & 0.115305 \\
\hline 580026 & 4.775 & 5.046661 & 5.216168 & 5.375807 \\
\hline 580025 & 2.825 & 1.627602 & 1.332668 & 0.922998 \\
\hline 580024 & 1,527 & 0.471913 & 0.318705 & 0.018573 \\
\hline 580023 & 2.53 & 0.764304 & 0.514835 & 0.007447 \\
\hline 580022 & 3.872 & 1.94822 & 1.738877 & 1.221415 \\
\hline 580021 & 5.198 & 2.252115 & 2.173053 & 1.499983 \\
\hline 580018 & 5.616 & 1.732888 & 1.14322 & 0.0454 \\
\hline 580017 & 5.854 & 1.815017 & 1.217213 & 0.060069 \\
\hline 580016 & 4.331 & 1.393305 & 0.954057 & 0.086036 \\
\hline 580015 & 7.438 & 3.831187 & 3.5234 & 2.528462 \\
\hline 580014 & 3.853 & 1.158984 & 0.772593 & 0.006107 \\
\hline 580013 & 2.655 & 1.801605 & 1.381086 & 1.096866 \\
\hline 580012 & 37.816 & 34.10225 & 33.59993 & 32.9285 \\
\hline 580011 & 8.505 & 8.319058 & 8.392523 & 7.951577 \\
\hline 580010 & 3.597 & 3.038113 & 2.865543 & 2.763409 \\
\hline 580009 & 18.023 & 17.46211 & 17.83424 & 17.74383 \\
\hline 580008 & 2.677 & 2.068681 & 2.198701 & 1.944177 \\
\hline 580007 & 2.689 & 1.797156 & 1.632051 & 1.358272 \\
\hline 580006 & 3.485 & 2.555585 & 2.595487 & 2.432724 \\
\hline 580005 & 9.422 & 10.19936 & 9.616998 & 9.577998 \\
\hline 580004 & 1.658 & 0.866497 & 0.781409 & 0.546919 \\
\hline 580003 & 1.446 & 1.110331 & 1.053102 & 0.966973 \\
\hline 580002 & 1.145 & 0.734778 & 0.69011 & 0.562863 \\
\hline 580001 & 0.7 & 0.545353 & 0.370943 & 0.294339 \\
\hline 580000 & 1.741 & 0.643079 & 0.464298 & 0.139744 \\
\hline 38003 & 3.55 & 1.183975 & 0.787549 & 0.103788 \\
\hline 38001 & 0.965 & 0.852782 & 0.751849 & 0.701565 \\
\hline 31005 & 5.2 & 1.699237 & 1.180749 & 0.151878 \\
\hline 30001 & 2.394 & 2.203681 & 2.249159 & 2.166924 \\
\hline
\end{tabular}


Table 4. The results of N IG-EGARCH pricing model.

\begin{tabular}{|c|c|c|c|c|}
\hline ‘code & real & Levy_GARCH & Levy & BS \\
\hline 580999 & 0.698 & O.50281S & 0.556481 & 0.525278 \\
\hline 580997 & 0.475 & 0.143068 & 0.100026 & 0.008904 \\
\hline 580996 & 0.915 & 0.609608 & 1.220966 & 1.303878 \\
\hline 580995 & 0.574 & $0.219 \mathrm{SS} 6$ & 0.401638 & 0.37326 \\
\hline 580994 & 1.281 & 0.904986 & 0.660899 & 0.484467 \\
\hline 580993 & 1.371 & O.S80422 & 0.684944 & 0.563156 \\
\hline 580992 & 0.642 & 0.257669 & 0.329755 & 0.240395 \\
\hline 580991 & 0.72 & 0.645687 & 0.632059 & 0.625238 \\
\hline 580990 & 1.073 & $0.40 \mathrm{SSS} 2$ & 0.396746 & 0.231048 \\
\hline 580989 & 1.526 & 0.705347 & 0.401881 & 0.115305 \\
\hline 580026 & 4.775 & 8.034697 & 5.216168 & 5.375807 \\
\hline 580025 & 2.825 & 1.444039 & 1.332668 & 0.922998 \\
\hline 580024 & I .527 & 0.475248 & 0.318705 & 0.018573 \\
\hline $5 \$ 0023$ & 2.53 & 0.785751 & 0.514835 & 0.007447 \\
\hline 580022 & 3.872 & 2.401636 & $1.738 \$ 77$ & 1.221415 \\
\hline 580021 & 5.198 & 7.776946 & 2.173053 & 1.499983 \\
\hline 580018 & 5.616 & 1.977906 & 1.14322 & 0.0454 \\
\hline 580017 & 5.854 & 1.826584 & 1.217213 & 0.060069 \\
\hline 580016 & 4.331 & 1.495201 & 0.954057 & 0.086036 \\
\hline 580015 & 7.438 & 5.208489 & 3.5234 & 2.528462 \\
\hline 580014 & 3.853 & 1.159478 & 0.772593 & 0.006107 \\
\hline 580013 & 2.655 & 2.013273 & 1.381086 & 1.096866 \\
\hline 580012 & 37.816 & 37.05279 & 33.59993 & 32.9285 \\
\hline 580011 & $8.50 \mathrm{~S}$ & 8.495398 & 8.392523 & 7.951577 \\
\hline 580010 & 3.597 & 4.399442 & 2.865543 & 2.763409 \\
\hline 580009 & 18.023 & 19.89462 & 17.83424 & 17.74383 \\
\hline 580008 & 2.677 & 2.850941 & 2.198701 & 1.944177 \\
\hline 580007 & 2.689 & 1.974428 & 1.632051 & 1.358272 \\
\hline 580006 & $3.4 \$ 5$ & 3.447789 & 2.595487 & 2.432724 \\
\hline 580005 & 9.422 & 11.0847 & 9.61 b998 & 9.577998 \\
\hline 580004 & 1.658 & 0.922121 & 0.781409 & 0.546919 \\
\hline 580003 & 1.446 & 1.136059 & 1.053102 & 0.966973 \\
\hline 580002 & 1.145 & 0.961087 & 0.69011 & 0.562863 \\
\hline 580001 & 0.7 & 0.32228 & 0.370943 & 0.294339 \\
\hline 580000 & 1.741 & 0.599363 & 0.464298 & 0.139744 \\
\hline 38003 & 3.55 & 1.088243 & $0.7 \mathrm{~s} 7 \mathrm{~s} 49$ & 0.103788 \\
\hline 38001 & 0.965 & 0.648044 & 0.751849 & 0.701565 \\
\hline 31005 & 5.2 & 1.923272 & 1.180749 & 0.151878 \\
\hline 30001 & 2.394 & 2.38783 & 2.249159 & 2.166924 \\
\hline
\end{tabular}


136 Li Zhou et al: : Empirical Research on Chinese Warrants Market Based on the Montecarlo Pricing Options Under Levy Process

Table 5. The results of VG-GJR-GARCH pricing model.

\begin{tabular}{|c|c|c|c|c|}
\hline `code & real & Levy_GARCH & Levy & BS \\
\hline 580999 & 0.698 & 0.636489 & 0.551678 & 0.525278 \\
\hline 580997 & 0.475 & 0.151371 & 0.100528 & 0.008904 \\
\hline 580996 & 0.915 & 1.30365 & 1.243136 & 1.303878 \\
\hline 580995 & 0.574 & 0.431629 & 0.424646 & $0: 37326$ \\
\hline 580994 & 1.281 & 0.74395 & 0.649486 & 0.484467 \\
\hline 580993 & 1.371 & 0.694297 & 0.74356 & 0.563156 \\
\hline 580992 & 0.642 & 0.318788 & 0.311715 & 0.240395 \\
\hline 580991 & 0.72 & 0.688944 & 0.659838 & 0.625238 \\
\hline 580990 & 1.073 & 0.490567 & 0.408242 & 0.231048 \\
\hline 580989 & 1.526 & 0.703569 & 0.402107 & 0.115305 \\
\hline 580026 & 4.775 & 5.054122 & 5.248217 & 5.375807 \\
\hline 580025 & 2.825 & 1.616516 & 1.331321 & 0.922998 \\
\hline 580024 & 1.527 & 0.472097 & 0.319042 & 0.018573 \\
\hline $5 \$ 0023$ & 2.53 & 0.771973 & 0.506973 & 0.007447 \\
\hline 580022 & 3.872 & 1.959845 & 1.745328 & 1.221415 \\
\hline 580021 & 5.198 & 2.354863 & 2.160318 & 1.499983 \\
\hline 580018 & 5.616 & 1.774358 & 1.139765 & 0.0454 \\
\hline 580017 & 5.854 & 1.807193 & 1.208279 & 0.060069 \\
\hline 580016 & 4.331 & 1.363859 & 0.96653 & 0.086036 \\
\hline 580015 & 7.438 & 3.942402 & 3.523871 & 2.528462 \\
\hline 580014 & 3.853 & 1.158308 & 0.773903 & 0.006107 \\
\hline 580013 & 2.655 & 1.647896 & 1.480369 & 1.096866 \\
\hline 580012 & 37.816 & 34.77012 & 34.24694 & 32.9285 \\
\hline 580011 & 8.505 & 8.089872 & 8.123394 & 7.951577 \\
\hline 580010 & 3.597 & 3.055949 & 2.882173 & 2.763409 \\
\hline 580009 & 18.023 & 17.56393 & 17.82058 & 17.74383 \\
\hline 580008 & 2.677 & 2.069457 & 2.029275 & 1.944177 \\
\hline 580007 & 2.689 & 1.771705 & 1.595787 & 1.358272 \\
\hline 580006 & 3.485 & 2.474702 & 2.723828 & 2.432724 \\
\hline 580005 & 9.422 & 9.964977 & 9.837742 & 9.577998 \\
\hline 580004 & 1.658 & 0.836846 & 0.768072 & 0.546919 \\
\hline 580003 & $1: 446$ & 1.034872 & i .056998 & 0.966973 \\
\hline 580002 & 1.145 & 0.822959 & 0.699987 & 0.562863 \\
\hline 580001 & 0.7 & 0.393372 & 0.350005 & 0.294339 \\
\hline 580000 & 1.741 & 0.654741 & 0.457789 & 0.139744 \\
\hline 38003 & 3.55 & 1.162806 & 0.791752 & 0.103788 \\
\hline 38001 & 0.965 & 0.819705 & 0.789201 & 0.701565 \\
\hline 31005 & 5.2 & 1.697384 & 1.116622 & 0.151878 \\
\hline 30001 & 2.394 & 2.158149 & 2.196378 & 2.166924 \\
\hline
\end{tabular}


Table 6. The results of VG-EGARCH pricing model.

\begin{tabular}{|c|c|c|c|c|}
\hline code & real & Levy_GARCH & Levy & BS \\
\hline 580999 & 0.698 & 0.610768 & 0.551678 & 0.525278 \\
\hline 580997 & 0.475 & 0.143131 & 0.100528 & 0.008904 \\
\hline 580996 & 0.915 & 0.639866 & 1.243136 & 1.303878 \\
\hline 580995 & 0.574 & 0.220068 & 0.424646 & 0.37326 \\
\hline 580994 & 1.281 & 0.919147 & 0.649486 & 0.484467 \\
\hline 580993 & 1.371 & 0.613497 & 0.74356 & 0.563156 \\
\hline 580992 & 0.642 & 0.247962 & $0.311 ? 15$ & 0.240395 \\
\hline 580991 & 0.72 & 0.631645 & 0.659838 & 0.625238 \\
\hline 580990 & 1.073 & 0.401689 & 0.408242 & 0.231048 \\
\hline 580989 & 1.526 & 0.696699 & 0.402107 & 0.115305 \\
\hline 580026 & 4.775 & 7.968 .543 & 5.24821 .7 & 5.375807 \\
\hline 580025 & 2.825 & 1.439972 & 1.331321 & 0.922998 \\
\hline 580024 & 1.527 & 0.480703 & 0.319042 & 0.018573 \\
\hline $5 \$ 0023$ & 2.53 & 0.800156 & 0.506973 & 0.007447 \\
\hline 580022 & 3.872 & 2.40137 & 1.745328 & 1.221415 \\
\hline 580021 & 5.198 & 7.683256 & 2.160318 & 1.499983 \\
\hline 580018 & 5.616 & 1.92982 & 1.139765 & 0.0454 \\
\hline 580017 & 5.854 & 1.806671 & 1.208279 & 0.060069 \\
\hline 580016 & 4.331 & 1.508436 & 0.96653 & 0.086036 \\
\hline 580015 & 7.438 & 4.843343 & $3.523 \$ 71$ & 2.528462 \\
\hline 580014 & 3.853 & 1.159126 & 0.773903 & 0.006107 \\
\hline 580013 & 2.655 & 1.913894 & 1.480369 & 1.096866 \\
\hline 580012 & 37.816 & 36.3972 & 34.24694 & 32.9285 \\
\hline 580011 & 8.505 & 8.762678 & 8.123394 & 7.951577 \\
\hline 580010 & 3.597 & 4.323088 & 2.882173 & 2.763409 \\
\hline 580009 & 18.023 & 19.90531 & 17.82058 & 17.74383 \\
\hline 580008 & 2.677 & $2 . . \$ 21059$ & 2.029275 & 1.944177 \\
\hline 580007 & 2.689 & 1.945918 & 1.595787 & 1.358272 \\
\hline 580006 & 3.485 & 3.524644 & 2.723828 & 2.432724 \\
\hline 580005 & 9.422 & 10.91313 & 9.837742 & 9.577998 \\
\hline 580004 & 1.658 & 0.92747 & 0.768072 & 0.546919 \\
\hline 580003 & 1.446 & 1.215662 & 1.056998 & 0.966973 \\
\hline 580002 & 1.145 & 0.95123 & 0.699987 & 0.562863 \\
\hline 580001 & 0.7 & 0.319569 & 0.350005 & 0.294339 \\
\hline 580000 & 1.741 & 0.583341 & 0.457789 & 0.139744 \\
\hline 38003 & 3.55 & 1.083989 & 0.791752 & 0.103788 \\
\hline 38001 & 0.965 & 0.617607 & 0.789201 & 0.701565 \\
\hline 31005 & 5.2 & 1.997062 & 1.116622 & 0.151878 \\
\hline 30001 & 2.394 & 2.691248 & 2.196378 & 2.166924 \\
\hline
\end{tabular}

\section{References}

[1] Box G, Mervin E. Muller. A note on the generation of random normal deviates[J]. The Annals of Mathematical Statistics, 1958, 29(2): 610-611.

[2] Boyle P. Options: A Monte Carlo approach[J]. Journal of Financial Economics, 1977, 4(3):323-338.

[3] Broadie M, Yamamoto Y. Application of the fast Gauss transform to option pricing[J]. Managment Science, 2003, 49(8): 1071-1088.

[4] Broadie M, Yamamoto Y. A double-exponential fast Gauss transform for pricing discrete pathdependent options[J]. Operations Research, 2005, 53(5): 764-779.

[5] Byun SJ, Min B. Conditional volatility and the GARCH option pricing model with non-normal innovations[J]. 3ournal of Futures Market, 2413, 33(1): 1-28.
[6] Carr P, Madan D B. Option valuation using the fast Fourier transform[J] Journal of Computational Finance, 1999, 2(4): 61-73.

[7] Carr P, Geman H, Madan D H and Yor M. The fine structure of asset returns: an empirical investigation[J]. Journal of Business, 2002, 75(2): 305-332.

[8] Carr P and Wu L R. The finite moment log stable process and option pricing[J]. Journal of Finance, 2003, 58(2): 753-777.

[9] Carriere J F. Valuation of the early exercise price for options using simulations and nonparametric regression[J]. Insurance: Mathematics and Economics, 1996, 19(1): 19-30;

[10] Carrosco M, Chernov M, Florens JP, Ghysels. Efficient estimation of general dynamic models with a continuum of moment conditions[J]. Journal of Econometrics, 2007, 140(2): 529-573.

[11] Chen Z, Feng L and Lin X. Simulating Levy process from their characteristic functions and financial applications[J]. ACM Transactions on Modeling and Computer Simulation, 2012, 22(3). 